\title{
SPATIAL INHIBITION OF RETURN PROMOTES CHANGES IN RESPONSE-RELATED MU AND BETA OSCILLATORY PATTERNS
}

\author{
Authors: E. Amenedo, E., Gutiérrez-Domínguez, F-J., Darriba, Á and Pazo-Álvarez, P
}

This is the peer reviewed version of the following article: E. Amenedo, E., Gutiérrez-Domínguez, F-J., Darriba, Á and Pazo-Álvarez, P (2015). Spatial Inhibition of Return promotes changes in response-related $\mathrm{mu}$ and beta oscillatory patterns. Neuroscience, 310, 616-628 doi: 10.1016/j.neuroscience.2015.09.072

This article may be used for non-commercial purposes in accordance with Elsevier and Pergamon terms and conditions for use of self-archived versions. 


\section{Spatial inhibition of return promotes changes in}

Response-related mu and beta oscillatory patterns

Authors: E. Amenedo*, E., Gutiérrez-Domínguez, F-J., Darriba, Á and Pazo-Álvarez, P

Department of Clinical Psychology and Psychobiology, Faculty of Psychology, University of Santiago de Compostela, Spain

*Corresponding author. Address: Department of Clinical Psychology and Psychobiology, Faculty of Psychology, Rúa Xosé María Suárez Núñez, S/N, 15782 Campus Vida, University of Santiago de Compostela, Spain. 


\begin{abstract}
The possible role that response processes play in Inhibition of Return (IOR), traditionally associated with reduced or inhibited attentional processing of spatially cued target stimuli presented at cue-target intervals longer than $300 \mathrm{~ms}$, is still under debate. Previous psychophysiological studies on response-related Electroencephalographic (EEG) activity and IOR have found divergent results. Considering that the ability to optimize our behavior not only resides in our capacity to inhibit the focus of attention from irrelevant information but also to inhibit or reduce motor activation associated with responses to that information, it is conceivable that response processes are also affected by IOR. In the present study, time-frequency (T-F) analyses were performed on EEG oscillatory activity between 2 and $40 \mathrm{~Hz}$ to check whether spatial IOR affects response preparation and execution during a visuospatial attention task. To avoid possible spatial stimulus-response compatibility effects and their interaction with the IOR effects, the stimuli were presented along the vertical meridian of the visual field.

The results differed between lower and upper visual fields. In the lower visual field spatial IOR was related to a synchronization in the pre-movement mu band at bilateral precentral and central electrodes, and in the post-movement beta band at contralateral precentral and central electrodes, which may be associated with an attention-driven reduction of somatomotor processing prior to the execution of responses to relevant stimuli presented at previously cued locations followed by a post-movement deactivation of motor areas. In the upper visual field, spatial IOR was associated with a decrease in desynchronization around response execution in the beta band at contralateral postcentral electrodes that might indicate a late (last moment) reduction of motor activation when responding to spatially cued targets. The present results suggest that different response processes are affected by spatial IOR depending on the visual field where the target is presented. 2015 IBRO. Published by
\end{abstract}

Key words: EEG oscillatory changes, spatial IOR, response preparation and execution, mu band, beta band, vertical asymmetries 


\section{Introduction}

The limited capacity of human brain makes it impossible to incorporate all available information. Visuospatial attention studies have shown that attention-shifting processes provide adaptive benefits by allowing the brain to select the information relevant in each moment as a basis for other processes, such as memory, learning and decision-making, which are essential for adaptation and correct functioning in everyday life. In this context, Posner and Cohen (1984) found that when responding to targets previously signaled by a peripheral cue two possible effects are observed on reaction time (RT) depending on the time interval between the cue and the target presentation. When that time is shorter than $250-300 \mathrm{~ms}$, RTs are faster (facilitation effect). At longer time intervals, however, RTs are slower. The authors explained this increase in RT as a mechanism that helps in selecting relevant information units by inhibiting attention from focusing on previously explored locations when there is enough time to process them. Posner et al. (1985) retrospectively named this mechanism Inhibition of Return (IOR).

Since its discovery, IOR has been observed in a wide variety of experimental situations within the visual, auditory, and tactile modalities (e.g., Spence et al., 2000). IOR has also been observed across a variety of tasks, including detection, localization, and discrimination tasks, and even in natural scenes (see Klein, 2000, for a review). However, at present no consensus has yet been reached regarding either the mechanisms of IOR or their functional significance.

The existing difficulty in characterizing the functional significance of IOR and its neural locus, led several research groups to examine the underlying electrophysiological mechanisms of behavioral IOR effects by means of event-related potential (ERP) analyses. Specifically, these ERP studies have mainly focused on stimulus-related components, and have shown that spatial IOR is frequently, but not always, associated with amplitude modulations in P1 and N1 targetlocked visual components. Such modulations have been generally interpreted as neural correlates of the effects of IOR on the perceptual-attentional processing of spatially cued target stimuli. Moreover, IOR effects on target-locked ERPs have resulted in other amplitude modulations in the ERP waveforms within latency intervals that do not coincide specifically with the peak of any component, and consisting in amplitude shifts whose functional interpretation is still under debate (see Gutiérrez-Domínguez et al., 2014).

However, the above-described IOR effects on target locked ERPs have not always been associated with behavioral IOR effects (i.e. slower RTs to cued targets; see for example Hopfinger and Mangun, 1998; McDonald et al., 1999; Doallo et al., 2004), suggesting that, possibly, response processing is also influenced by it (Kingstone and Pratt, 1999; Pasto" tter et al., 2008). Responselevel explanations of IOR have received support from behavioral evidence showing that it might be associated with a more conservative response criterion on cued trials (Ivanoff and Klein, 2001), and that IOR can affect oculomotor programing (Ro et al., 2000). To explore more directly the response-related processes affected by IOR, Prime and Ward $(2004,2006)$ measured the effects of IOR on the lateralized readiness potential (LRP), and examined the possibility that response related effects of IOR may arise at either decisional or motor stages of response processing. To that end, they examined the target-locked LRP (T-LRP) and the response-locked LRP (R-LRP) components starting from the premise that if IOR arises from inhibition of motor processes, then the interval between the onset of the R-LRP and the response should be longer under IOR, while if IOR arises from decisional but not motor processes, then only the interval between the target presentation and the response would be affected by IOR (affecting the latency of the T-LRP).

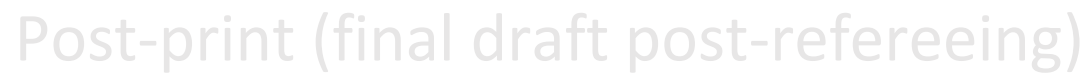


They found IOR effects on T-LRP latency but not on R-LRP latency, concluding that IOR may not be related to response preparation timing but only to pre-motor selection processes. More recently, Amenedo et al. (2014) examined the amplitude changes in R-LRP, and found that IOR was related to a significant amplitude reduction of this component when responding to previously cued targets, suggesting that response preparation could be affected when responding to targets presented at previously cued locations.

Event-related changes in Electroencephalographic (EEG) activity may be studied with different approaches. One of the most frequently employed has been the ERP technique, which is based on the measurement of amplitude changes in the ongoing EEG activity time locked to stimulus presentation or to response execution. An alternative and complementary approach is the measurement of event-related changes in frequency oscillations that occur in the ongoing EEG activity in association with stimulus presentation or response production. One of the most extended methods for the analysis of EEG oscillations is the so-called time-frequency (T-F) analysis that allows examination of the spatio-temporal changes in spectral power within different frequencies relative to a baseline period and related to stimulus or response processing (see Roach and Mathalon, 2008 for a comprehensive review on ERP and T-F methodologies).

In the context of movement execution, EEG activity within sensorimotor areas of the human brain has long been known to exhibit oscillatory behavior, which makes the T-F approach suitable for studying possible effects of IOR on response processes. Of particular interest have been oscillations within two specific frequency bands, the mu $(8-14 \mathrm{~Hz})$ and beta $(15-30 \mathrm{~Hz})$ bands, as they have been shown to be modulated during and following the preparation and performance of voluntary movements (Salmelin et al., 1995; Pfurtscheller et al., 1996a,b; Leocani et al., 1997; Cassim et al., 2001; Jurkiewicz et al., 2006; Parkes et al., 2006), passive movements (Cassim et al., 2001), imagined movement (Pfurtscheller et al., 2005, 2006), and even tactile stimulation (Neuper and Pfurtscheller, 2001; Cheyne et al., 2003; Gaetz and Cheyne, 2006). Modulation of the mu and beta band oscillations that accompany voluntary movements has been described and takes one of two forms. Beginning as early as $2 \mathrm{~s}$ prior to movement initiation (Pfurtscheller and Berghold, 1989; Leocani et al., 1997), a reduction in power in both the mu and beta frequency bands, known as event-related desynchronization (ERD), has been observed over sensorimotor areas with a contralateral predominance in the case of the beta band (see Pfurtscheller and Lopes da Silva, 1999 for review) and more bilateral for the mu band (Salmelin et al., 1995) although contralateral predominance has also been shown in this frequency band (see Pfurtscheller et al., 1996a, for review). Desynchronization of these oscillations, the result of asynchronous activity within these cortical networks, has been related to neural activation (Pfurtscheller and Berghold, 1989). Following movement termination, while mu power returns slowly to baseline (Salmelin and Hari, 1994; Salmelin et al., 1995; Leocani et al., 1997), beta power consistently returns to and exceeds premovement levels (Pfurtscheller et al., 1996a; Jurkiewicz et al., 2006). This event, known as event-related synchronization (ERS) begins within several hundred milliseconds of movement termination and persists for several hundred more. Although it is generally believed that ERS reflects a neural deactivation, the socalled 'idling' hypothesis (Pfurtscheller et al., 1996a; Cassim et al., 2001), this specific beta band ERS that follows movement termination is known as postmovement beta rebound (PMBR) and has been suggested to represent an inhibition of motor cortex (Salmelin et al., 1995; Jurkiewicz et al., 2006) or a sensorimotor reafference (Cassim et al., 2001) after movement execution. 
Exploring spatial IOR effects on response-related EEG oscillatory activity by means of T-F analyses, Pastotter et al. (2008) examined changes in ERS-ERD patterns (temporal spectral evolution analysis, Hari and Salmelin, 1997) restricted to the pre-movement $15-25 \mathrm{~Hz}$ beta band in two IOR designs: a target-target design, and a cue-target design. They found that in the targettarget design behavioral IOR was associated with an increase in contralateral beta ERS while in the cuetarget design IOR was related to a decrease in beta ERD. They concluded that IOR arises from the inhibition of motor processes affecting different mechanisms depending on the design employed. Specifically, the authors concluded that with the target-target design some kind of passive inhibition (idling state) would occur, while with the cue-target design an active inhibition would be associated with IOR.

Taking into account the previous findings, and considering that the ability to optimize our behavior not only resides in our capacity to inhibit the focus of attention from returning to previously explored locations but also to inhibit or reduce motor activation associated with responses to stimuli appearing at those locations, it is conceivable that response processes are also affected by IOR.

In this context, the present study aimed at exploring IOR effects on $\mathrm{T}-\mathrm{F}$ changes in the EEG activity in those oscillatory patterns that have been found to systematically react to movement processing and execution (Salmelin et al., 1995; Pfurtscheller et al., 1996a,b). To that end, we examined the oscillatory pattern of EEG activity under spatial IOR during the execution of a cueback visuospatial attention task (see Gutierrez-Domínguez et al., 2014). Due to the well established effects of stimulus-response spatial compatibility on response processing (see Hommel, 2011 for a review), and to the possible interactions between these effects and IOR effects (see Ivanoff et al., 2002 for a review) the stimuli were presented along the vertical axis of the visual field. Moreover, due to the frequently reported existence of visual asymmetries when the stimuli are presented along the vertical meridian (Rezec and Dobkins, 2004; Amenedo et al., 2007; Karim and Kojima, 2010; Thomas and Elias, 2011), and to previous results showing the existence of such asymmetries on the effects of spatial IOR on stimulus-locked and on responselocked ERPs (Amenedo et al., 2014; Gutiérrez-Domínguez et al., 2014) the data from each visual field were analyzed separately. With this design, we aimed at exploring IOR effects on EEG activity related to response processing by analyzing the spatial and temporal changes in frequency oscillations between 2 and $40 \mathrm{~Hz}$ related to response preparation and execution.

\section{Experimental procedures}

\section{Participants}

Seventeen young adults (10 females, $24.85 \pm 5.99$ years, range 19-37) participated in the study. All participants were healthy well functioning without a history of neurological or psychiatric disorders, had normal or corrected-to-normal visual acuity, reported normal color vision, and were right handed (Oldfield, 1971). Informed consent was obtained from all participants, and they received a monetary compensation for their participation.

\section{Stimuli and experimental design}

During the task (see Fig. 1), one central and two peripheral (external edge $4.5^{\circ}$ of visual angle from the center of the screen) light gray boxes (RGB $200,200,200,1.5^{\circ} \times 1.5^{\circ}$ of visual angle) were always present in the vertical meridian of a computer screen $(100-\mathrm{Hz}$ refresh rate). A central fixation cross (RGB $150,150,150,0.1^{\circ} \mathrm{x} 0.1^{\circ}$ of visual angle) was also present and participants 
were instructed to maintain their gaze on it during the task performance. Each trial began with a 1500-ms blank screen (RGB 50,50,50, average luminance $2.4 \mathrm{~cd} / \mathrm{m} 2$ ) that defined the background screen. After this, a blue (RGB 0,0,255, average luminance $8.3 \mathrm{~cd} / \mathrm{m} 2$ ) or red (RGB 175,0,0, average luminance $8.2 \mathrm{~cd} / \mathrm{m} 2$ ) patch was presented during $100 \mathrm{~ms}$ filling one of the two peripheral boxes ( 0.5 probability). This patch served as a cue for location or color dimension, and it was uninformative related to both location and color dimensions of the target. After a new blank screen of 500-ms duration, a cue-back consisting of a green patch (RGB 0,95,0, average luminance 8.4 $\mathrm{cd} / \mathrm{m} 2$ ) filling the central box was presented for $100 \mathrm{~ms}$. After another blank interval of $1300 \mathrm{~ms}$, a target was presented until response or a maximum of $1500 \mathrm{~ms}$. Target stimuli consisted of a blue or red patch ( 0.5 probability), identical to the cue, filling the lower or upper box $(0.5$ probability). Target shared color or location with the cue in $50 \%$ of trials. The duration of the interval between the cue onset and the target onset (see Fig. 1) defined a cue-target onset asynchrony (CTOA) of $2000 \mathrm{~ms}$. Four types of trials resulted from cue and target color and location combinations: trials with both location and color cued (cue and target appearing at the same location and with the same color), trials with only location cued (cue and target appearing at the same location but with different color), trials with only color cued (cue and target sharing color but appearing at different locations), and trials with neither location nor color cued (cue and target appearing at different locations and with different color).

Participants were sitting in an armchair placed at $112 \mathrm{~cm}$ distance from the computer screen; they were asked to respond to target color (red or blue) irrespective of its location by pressing a button (Response Box RB-834 model, Cedrus Corporation) with their right hand to one color and another button with their left hand to the other color while maintaining central fixation. Assignment of response hand to each color was counterbalanced across participants. Both speed and accuracy were stressed in the instructions (please see Gutiérrez-Domínguez et al., 2014, for a more complete description and justification of this discrimination task). To prevent the increased variability observed in scalp distribution of oscillatory brain activity associated with nondominant hand executions (Stanca' k and Pfurtscheller, 1996; Gaetz et al., 2010), only trials with responses executed with the dominant hand (right hand in our participants) were included in the statistical analyses. The task was divided into 25 blocks of 64 trials, mixing different trial conditions in each block randomly. There was a 3-s rest between each block. After that, participants could continue with the task as soon as they wanted by pressing a button of the response device. Due to previous results in our laboratory with the same task showing neither IOR effects of color cueing nor interactions between location and color cueing (see GutierrezDomínguez et al., 2014) we focus our analyses on the comparison between trials with neither location nor color cued (uncued condition thereafter) and trials with only location cued (locationcued condition thereafter).

\section{Recording and analysis}

\section{Behavioral data}

Reaction times (RTs) and accuracy (hits and errors) were on-line recorded for all participants under all conditions in all experimental blocks. Only RT values associated with correct responses were included in the analyses. Responses were considered correct when RTs were within \pm 3 standard deviations of the mean RT for each condition. Mean correct RTs and error rates (\%) were submitted to a repeated measures analysis of variance (ANOVA) with cueing (uncued vs. 
location-cued) as within-subject factor. Whenever appropriate, degrees of freedom were corrected by the conservative Greenhouse-Geisser estimate. An alpha level of .05 was used for all analyses.

\section{EEG recording}

Recordings were made in an electrical shielded and sound-attenuated room. Continuous EEG activity was recorded with a Brain Vision Recorder (Brain Products, Inc.) from 60 scalp Ag-AgCl electrodes according to the extended 10/20 International System (see Fig. 2). The cephalic electrodes were referred to the nose tip and grounded with an electrode placed at $10 \%$ of the nasion-inion distance above nasion. Vertical and horizontal electrooculogram (EOG) was recorded from above and below the participant's left eye and from the outer canthi of both eyes, respectively. Electrode impedances were kept below $10 \mathrm{k} \Omega$. Sampling rate was $500 \mathrm{~Hz} / \mathrm{channel}$. EEG signal was continuously amplified $(10 \mathrm{~K})$ and filtered online with a band pass of $0.01-100$ $\mathrm{Hz}$.

\section{EEG analysis}

Data from all conditions were epoched into segments of $4500 \mathrm{~ms}$ (-3500 ms to $1000 \mathrm{~ms}$ relative to button-press) and merged together for each subject. Prior to the spectral analyses, artifacts were removed using EEGLAB (Delorme and Makeig, 2004), a freely available open source software toolbox (Swartz Center for Computational Neurosciences, La Jolla, CA; http://www.scen. ucsd.edu/eeglab) running under Matlab (MathWorks, Inc, Natick, MA, USA) in the following way. First, epochs containing non-stereotyped artifacts (e.g. cable movement, swallowing) were manually removed whereas epochs containing repeatedly occurring, stereotyped artifacts (e.g., eye blinks, muscle artifact etc) were kept. Then, extended infomax independent component analysis(ICA, Bell and Sejnowski, 1995; Lee et al., 1999) was applied individually for each subject, using a weight change of $<10-^{7}$ or 512 interactions as a stop criterion. Component activations were subsequently assessed and categorized as brain activity or non-brain artifact (e.g., muscle, electrode artifact or eye movement activity) by visual inspection based on their scalp topographies, time courses, ERP-image, and activation spectra. Rejected ICAs comprised three main artifact categories: (A) Ocular artifacts were defined as smoothly decreasing EEG spectrum typical of an eye artifact and a scalp map showing a strong far-frontal projection. (B) Muscular artifacts were spatially localized, showing high power at high frequencies $(20-50 \mathrm{~Hz})$. (C) Electrode artifacts were characterized for their topography (affecting only one electrode) and ERP images showing non time-locked artifacts with generally large amplitudes.

After identification of components constituting artifacts, individual EEG data containing all conditions were reconstructed without those components. Finally, epochs corresponding to each experimental condition were extracted from ICA-pruned data for each subject.

In order to make comparisons between conditions, the number of trials was adjusted for each subject. Trials on those conditions were randomly selected. This procedure resulted in around 30 trials per condition for each subject on average.

\section{$T-F$ analysis}

ICA-corrected data from uncued conditions and locationcued conditions were submitted to trialby-trial T-F analysis using EEGLAB (Delorme and Makeig, 2004) running under Matlab (MathWorks, Inc., Natick, MA). Spectral changes in oscillatory activity were analyzed using a wavelet transform. T-F analysis was computed from- 3500 to 1000 ms relative to button press 
for every electrode, subject and condition separately using a Gaussian-windowed sinusoidal moving Morlet wavelet with linearly increased cycles from 2 cycles for the lowest frequency ( 2 $\mathrm{Hz}$ ) to 20 cycles for the highest frequency $(40 \mathrm{~Hz}$ ) analyzed (step size, $0.5 \mathrm{~Hz}$ ). Changes in eventrelated spectral power response at different time points, relative to power in baseline (from -3000 to -2500 ms), were computed by the Event-Related Spectral Perturbation (ERSP) index (Delorme and Makeig, 2004). The ERSP can be viewed as a generalization of the ERD. The ERSP measures average dynamic changes in amplitude of the broad band EEG frequency spectrum as a function of time relative to an experimental event. That is, the ERSP measures the average time course of relative changes in the spontaneous EEG amplitude spectrum induced by a set of similar experimental events. These spectral changes typically involve more than one frequency or frequency band; so full-spectrum ERSP analysis yields more information on brain dynamics than the narrow-band ERD. The ERPS are color-coded T-F images of mean log spectral differences that have been normalized by subtracting the mean log power spectrum within a defined baseline period (in our case -3000 to $-2500 \mathrm{~ms}$ ) (see Makeig and Onton, 2012). Once the spectral power changes as specified above were obtained, the differences between conditions in the resulting changes in power $(\mathrm{dB})$ were addressed using permutation analyses (2000 permutations, alpha level 0.05). The obtained power values across the frequency bands, time intervals, and electrodes where permutations showed significant differences were corrected for multiple comparisons with the false discovery rate (fdr). Moreover, the power values across the electrodes, the time intervals, and the frequency bands where these permutations with fdr correction showed significant differences between uncued and location-cued conditions were submitted to parametric analyses by means of separate repeated measures ANOVAs for each visual field with the within-subject factors location cueing (uncued vs. location-cued) and electrode (for lower visual field, LVF, analysis, mu band (9-11 Hz): F3, F1, Fz, F2, F4, F6, FC5, FC3, FC1, FCz, FC2, FC4, FC6, C3, C1, Cz, C2, C4, C6, FT8, T8; beta band (28-32 Hz): F7, F5, F3, F1, FC3, FC1, FCz, C3, C1, Cz, and for upper visual field, UVF, analysis, beta band (26-28 Hz): CP5, CP1, P7, P5, P3, P1, Pz, $\mathrm{PO} 7, \mathrm{PO} 3, \mathrm{POz}, \mathrm{O} 1)$. The level of significance was established in 0.05 .

\section{Results}

\section{Behavioral data}

The repeated measures ANOVAs showed significantly slower RTs to targets appearing at previously cued locations (Table 1) in both the $\operatorname{LVF}\left(\mathrm{F}(1,16)=4.14, \mathrm{p}<.05, \mathrm{~g}^{2}=.21\right)$ and the UVF $\left(\mathrm{F}(1,16)=13.6, \mathrm{p}<.01, \mathrm{~g}^{2}=.46\right)$, whereas there were no significant differences in error rates (Table 1) between uncued and location-cued conditions in both the $\operatorname{LVF}\left(\mathrm{F}(1,16)=1.11, \mathrm{p}=.3, \mathrm{~g}^{2}=.07\right)$ and the $\operatorname{UVF}\left(\mathrm{F}(1,16)=1.74, \mathrm{p}=.2, \mathrm{~g}^{2}=.1\right)$.

\section{EEG data. $T-F$ results}

Fig. 2 shows the ERSP image corresponding to power changes (dB) across time at C3 electrode under the uncued condition in the LVF. In this image, and in the corresponding topographical maps, with descriptive purposes, it can be seen the typical oscillatory pattern showing mu and beta changes associated with the execution of a voluntary movement, and described in previous literature (see, for example, Pfurtscheller et al., 1996b; Jurkiewicz et al., 2006). This pattern consisted in contralateral mu and beta desynchronization prior to movement, and in contralateral beta synchronization after movement. Fig. 3 shows the ERSP images corresponding to power $(\mathrm{dB})$ changes across time under location-cued and uncued conditions in both visual fields at the $\mathrm{C} 3$ electrode. The images show different patterns of event-related power changes in each visual 
field associated with spatial IOR. In the LVF, a different pattern of activation in mu and beta bands was observed between uncued and locationcued conditions, while in the UVF differences between cueing conditions were found only in the beta band. LVF. Mu band $(9-11 \mathrm{~Hz})$ The permutation analyses with fdr correction for multiple comparisons showed significant differences between cueing conditions from 9 to $11 \mathrm{~Hz}$ within a time interval from -240 to $-230 \mathrm{~ms}$ across bilateral precentral and central electrodes (see Fig. 3). The repeated measures ANOVAs on the power values within this frequency band, time interval, and group of electrodes showed that the differences were due to a power decrease relative to baseline (desynchronization) in uncued trials (mean power value $-.81 \pm .48 \mathrm{~dB}$ ) that was substituted by a power increase relative to baseline (synchronization) in location-cued trials (mean power value $.29 \mathrm{~dB} \pm .44 \mathrm{~dB})(\mathrm{F}(1,16)=8.47, \mathrm{p}<.01$, $\left.\mathrm{g}^{2}=.35\right)$. The factor electrode was significant $\left(\mathrm{F}(20,320)=1.9, \mathrm{p}<.01, \mathrm{~g}^{2}=.11\right)$ although the Bonferroni test for multiple comparisons failed to show any significant difference among them. No interaction was observed between cueing condition and electrode $(F(20,320)=.48, p=.1$, $\left.\mathrm{g}^{2}=.03\right)$.

\section{LVF. Beta band $(28-32 \mathrm{~Hz})$}

In the beta band, the permutation analyses with fdr correction showed differences in the LVF between uncued and location-cued trials from 28 to $32 \mathrm{~Hz}$ and from 145 to $155 \mathrm{~ms}$ post-movement across contralateral precentral and central electrodes (see Fig. 3). The repeated measures ANOVAs executed on these power values indicated that the differences were due to beta desynchronization (mean power value $-.59 \pm .19 \mathrm{~dB}$ ) in uncued trials that was substituted by beta synchronization (mean power value $.25 \pm .23 \mathrm{~dB})$ in location-cued trials $(\mathrm{F}(1,16)=15.74, \mathrm{p}<.001$, $\left.\mathrm{g}^{2}=.5\right)$. No differences among electrodes $\left(\mathrm{F}(9,144)=.35, \mathrm{p}=.76, \mathrm{~g}^{2}=.02\right.$, e $\left.=.29\right)$ nor interaction between cueing condition and electrode $\left(\mathrm{F}(9,144)=.49, \mathrm{p}=.67, \mathrm{~g}^{2}=.03, \mathrm{e}=.31\right)$ were observed.

\section{UVF. Beta band (26-28 Hz)}

In the UVF the pattern of results showed differences between uncued and location-cued conditions only in the beta band around response execution. Specifically, the permutation analyses with fdr correction showed significant differences in a frequency band from 26 to $28 \mathrm{~Hz}$, and from -50 to $20 \mathrm{~ms}$ in contralateral postcentral electrodes (see Fig. 3). The repeated measures ANOVA on the above power values indicated that spatial cueing was associated with a reduced beta band desynchronization $\left(\mathrm{F}(1,16)=18.08, \mathrm{p}<.001, \mathrm{~g}^{2}=.53\right)$ in location-cued trials (mean power value $-.2 \pm .18 \mathrm{~dB}$ ) compared to uncued trials (mean power value $-1.13 \pm .2 \mathrm{~dB}$ ). No differences were observed among electrodes $\left(\mathrm{F}(10,160)=.13, \mathrm{p}=.94, \mathrm{~g}^{2}=.01\right.$, $\left.\mathrm{e}=.36\right)$, and no interaction was found between cueing condition and electrode $\left(\mathrm{F}(10,160)=.28, \mathrm{p}=.84, \mathrm{~g}^{2}=.02\right.$, e =.37).

\section{Discussion}

In the present study, the main objective was to analyze the effects of spatial IOR on behavioral execution and on oscillatory EEG activity related to response preparation and production in healthy young adults. For that purpose, RTs, error rates, and EEG activity were obtained during a cue-back visuospatial attention task in which the target stimulus could appear at uninformative cued or uncued locations while participants were required to give a two-choice discrimination response to the color of the target stimulus (blue or red) and to ignore its spatial location. To control for possible interactions between IOR and spatial compatibility between target location and response hand, the stimuli were presented along the vertical axis. Moreover, as vertical 
asymmetries in visual processing have been reported in behavioral and psychophysiological studies, we analyzed the data from the lower and the upper visual fields separately.

\section{Behavioral execution}

The present results, in agreement with previous studies in the context of IOR research, showed that spatial cueing was associated with a significant slowing of RTs when the target was spatially signaled by an uninformative peripheral cue in both visual fields. Moreover, this increase of RT was accompanied by an absence of differences in error rates, which indicates that the accuracy of execution was not affected by spatial cueing, as it is also frequently observed in this research context (see, for review, Klein, 2000; Samuel and Kat, 2003; Lupiáñez et al., 2006).

\section{EEG results}

The $\mathrm{T}-\mathrm{F}$ analyses showed that spatial cueing was associated with changes in the oscillatory pattern related to response production that differed depending on the visual field. In the LVF, a shift from desynchronization to synchronization was observed under IOR in bilateral premovement mu band and in contralateral post-movement beta band. In the UVF, a decrease in desynchronization in contralateral beta band was observed during response execution.

\section{LVF. Mu $(9-11 \mathrm{~Hz})$ and beta $(28-32 \mathrm{~Hz})$ bands}

When targets were presented in the LVF, differences between uncued and location-cued trials were observed in the mu and beta frequency bands as explained and discussed in the paragraphs below.

Mu band $(9-11 \mathrm{~Hz})$. In the mu band, differences consisted in a shift from desynchronization (uncued trials) to synchronization (location-cued trials) in the premovement interval with a bilateral frontal and central scalp distribution. Specifically, location cueing promoting behavioral IOR was associated with EEG synchronization between 9 and $11 \mathrm{~Hz}$ from -240 to $-230 \mathrm{~ms}$ relative to button press.

$\mathrm{Mu}$ rhythms are typically identified as $8-14-\mathrm{Hz}$ oscillations that are maximal over the sensorimotor cortex when the individual is at rest. It has been frequently observed that within approximately two seconds before voluntary movements or somatosensory stimulation, these rhythms desynchronize and attenuate over contralateral or bilateral somatosensory areas (Pfurtscheller et al., 1994, 1996b; Salmelin et al., 1995; Pineda, 2005). This desynchronization of mu rhythms has been generally interpreted as an index of sensorimotor activation needed to prepare and execute a motor command leading to any movement (Pfurtscheller et al., 1994; Salmelin et al., 1995).

On the other hand, the synchronization of mu rhythms during the absence of movement or somatosensory stimulation has been generally interpreted as an 'idling' or 'nil working' state of sensorimotor areas when no further motor commands are sent to the muscles controlling the movement and no more afferent input from the somatosensory receptors reach the somatosensory cortex (Pfurtscheller et al., 1996b, 1997; Salmelin et al., 1995).

EEG studies have identified mu desynchronization during finger movement to be maximal over the contralateral sensorimotor cortex (Pfurtscheller et al., 1996a; Leocani et al., 1997; Cassim et al., 2001). Further studies have revealed that the location of mu band synchronization is slightly more posterior than the mu desynchronization, suggesting that the mu rhythm is generated mainly 
in the post-Rolandic somatosensory area (Pfurtscheller et al., 1996b; Jurkiewicz et al., 2006). A similar study employing magnetoencephalography (MEG) also found mu rhythm generators extended well into the postcentral cortex and bilaterally located aroundhand areas (Salmelin et al., 1995; Jurkiewicz et al., 2006).

The most typical task situation employed to measure these rhythms, and from which their functional interpretation has been derived, was to instruct subjects to execute or imagine (Pfurtscheller et al., 2006) selfpaced movements, or to execute simple stimulus-driven movements (Pfurtscheller et al., 1994).

More recently, alternative interpretations of mu synchronization prior to movement have been offered from studies employing more complex go-nogo tasks (Mazaheri et al., 2009) and motor learning tasks (Hummel et al., 2002). Employing a go-nogo task, Mazaheri et al. (2009) have related mu synchronization under movement withholding in nogo trials to an automatic, overlearned response mode where cortical areas are less active to inputs from other regions such as signals serving to inhibit an automatic motor action (Mazaheri et al., 2009). Hummel et al. (2002) with a task where subjects were instructed to withhold motor responses of previously learned motor programs, interpreted the observed increase in mu power associated with response withholding as a context-dependent inhibitory control of acquired motor programs.

However, in all the above-cited studies, the synchronization in mu band was always observed prior to movement withholding (i.e. in the absence of movement). In the present study, however, premovement mu synchronization was observed prior to the execution of correct responses to target visual stimuli appearing at previously cued locations (i.e. associated with overt movements). Therefore, it is difficult to interpret the result on the IOR-related mu synchronization observed in the present study as reflecting an idling state of the sensorimotor areas, or an inhibition of motor commands, because in doing such interpretation we would strictly mean that an effective and observable movement was executed under the absence of sensorimotor afferences and motor commands in the corresponding somatosensory cortical areas.

A possible explanation to the present results on mu band comes from studies in the context of spatial attention employing somatosensory stimulation tasks (Jones et al., 2010; Anderson and Ding, 2011; Haegens et al., 2011). These studies have generally found mu desynchronization to attended tactile stimulation and mu synchronization to ignored tactile stimulation. Specifically, Jones et al. (2010) employing a cued spatial attention task instructed participants to attend to visual-auditory cues signaling either to attend to pulse stimuli delivered to hand, foot, or both while recording MEG activity. They found that cued attention affected prestimulus mu and beta rhythms in somatosensory hand areas by desynchronizing them to attended hand stimulation (attend-in condition in their study), and synchronizing them to attended foot stimulation (attendout condition in their study). They also found that tactile detection probabilities decreased linearly with prestimulus mu and beta power. The authors interpreted their results as implying that the reduction of mu and beta rhythms with cued attention to tactile-stimulated areas is an active cortical gating mechanism that increases the perceptual salience of those attended tactile stimuli. Haegens et al. (2011), also employing MEG recordings, investigated whether prestimulus mu activity reflects the allocation of attentional resources in the somatosensory system. Specifically, they instructed subjects to discriminate between variably predictive $(100 \%, 75 \%, 50 \%)$ cued tactile stimulation delivered to either their left or right hand. Their results showed that prestimulus mu power lateralization varied as a function of cue predictability in such a way that it 
was strongly lateralized with fully predictive cues (100\%), less lateralized with lower cue reliability (75\%) and bilateral with no predictive cues $(50 \%)$. They concluded that their results on mu extended the notion that alpha band activity is focal and functionally specific to the sensory area in question, and that it is involved, by top-down attentional control, in shaping the functional state of the working brain by determining the functional engagement and disengagement of specific regions by power decreases in task-relevant areas and power increases in task-irrelevant areas. Anderson and Ding (2011) recorded EEG during a somatosensory oddball task where subjects had to fixate in the center of a computer screen and direct their attention to either their right, left or both hands while standard (80-92\%) or target (8-20\%) tactile stimuli were delivered randomly and equiprobably to their right or left median nerve. They found significant decreases in prestimulus mu power in the primary somatosensory cortex (SI; source level analyses) in the attend conditions compared to the ignore conditions. Anderson and Ding (2011) interpreted their results as a top-down modulation of prestimulus mu power resulting from the direction of spatial attention in anticipation of the upcoming stimulus.

Previous ERP results in our laboratory with the same task employed in the present experiment have shown IOR-related reductions in the amplitude of R-LRP also in the LVF that have been related to reduced/inhibited response preparation to spatially cued targets (see Amenedo et al., 2014). Taking into account the above studies in the context of somatosensory spatial attention and mu modulations, and our previous response-locked ERP results, the synchronization of preresponse mu rhythm observed in the present study under spatial IOR could be related to an attention-driven reduction of somatomotor processing that leads to power increases prior to the execution of responses to target stimuli that are presented at previously cued locations and whose visual processing has been reduced (see also Gutiérrez- Domínguez et al., 2014).

Beta band $(28-32 \mathrm{~Hz})$. In the beta band, a contralateral shift from desynchronization (uncued trials) to synchronization (location-cued trials) was observed in the LVF after response execution. Specifically, location cueing promoting behavioral IOR was associated with EEG synchronization between 28 and $32 \mathrm{~Hz}$, and from 145 to $155 \mathrm{~ms}$ post-movement across contralateral precentral and central electrodes.

Beta rhythms have been more frequently studied than mu rhythms in association with movement preparation and execution. Beta desynchronization during finger movement has been found in EEG studies to be maximal over the contralateral sensorimotor cortex, with a similar contralateral sensorimotor cortical location for maximal beta synchronization following movement termination (Pfurtscheller et al., 1996a; Leocani et al., 1997; Cassim et al., 2001). Moreover, it has been observed in both EEG and MEG studies that the location of beta band synchronization is slightly more anterior to the beta desynchronization, suggesting that at least some components of the beta rhythm are generated in the anterior bank of the central sulcus, in the pre-Rolandic motor area (Pfurtscheller et al., 1996b; Jurkiewicz et al., 2006, but see Parkes et al., 2006). Taking the above into account, post-movement synchronization within the beta band is generally thought to reflect the deactivation or 'idling' of motor cortical networks (Pfurtscheller, 2001), or the resetting of previously activated motor networks (Jurkiewicz et al., 2006; Pfurtscheller et al., 2003) once the movement has been executed.

Post-movement beta synchronization has also been observed after motor imagery and medial nerve stimulation (Neuper and Pfurtscheller, 2001). Moreover, Pogosyan et al. (2009) with the objective of examining whether beta activity is actively involved in human motor behavior or is 
merely associated with it as an epiphenomenon, employed transcranial alternating-current stimulation at $20 \mathrm{~Hz}$ while subjects performed a tracking task in which they had to track the movement of a visual target. They found that this stimulation synchronized beta activity in sensorimotor areas and slowed voluntary movements in healthy participants. The authors interpreted the irresults as providing evidence of a causal link between beta synchronization and slowing of voluntary movements. More recently, an association of beta oscillations with inhibitory control was suggested. Specifically, intracranial measurements exhibited increased beta band activity and interregional coherence in the right inferior frontal gyrus and preSMA with successful stop trials (Swann et al., 2009, 2012; Aron, 2011; Huster et al., 2013).

Considering the above findings and the functional role given to beta synchronization, the IOR effects observed in the present study in post-response beta band could be interpreted as a deactivation of motor areas after responding to targets presented at previously cued locations.

In a previous study within the context of visual attention, Pastotter et al. (2008) studied the possible involvement of response inhibition in spatial IOR by employing two task designs: a cuetarget design, and a target-target design. With these two tasks they analyzed beta band power changes by means of the eventrelated desynchronization/synchronization (ERD/ERS) index within 15-25 Hz (temporal spectral evolution analysis, Hari and Salmelin, 1997). The authors started from the premise that in the classical cue-target design a response to the cue must be inhibited, and this inhibition may still be present when the target appears confounding the genuine IOR effects. In the target-target design, by contrast, all stimuli require a response, and every target works as a cue for the following in the task sequence so no response withholding is necessary, and only IOR effects remain. In both tasks, with a vertical presentation of stimuli, participants were required to fixate on the center of a computer screen and to make a location discrimination response by pressing a left key with their left index finger to targets presented 6.5- above fixation, and a right key with their right index finger to targets presented 6.5- below fixation. They found that in the cue-target design IOR was associated with a decrease in beta ERD, suggesting an active inhibition of motor responses to the cued targets, while in the target-target design IOR was related to an increase in beta ERS suggesting a passive inhibition of motor activity after response execution. Based on their review of previous literature on movement-related beta oscillations, Pastótter et al. (2008) interpreted their results as showing that response inhibition processes are involved in IOR but in a different manner depending on the design.

In the present study, a cue-back design with stimuli presented along the vertical axis was used. As Pastótter et al. (2008) pointed out, cue-target designs have been the most frequently used to study IOR. Beyond the fact that response inhibition to the cue could be present when the target appears, these cue-target designs have been put into question because they do not assure that the attentional focus has been disengaged from the cue at the time of target presentation (Prime et al., 2006) ana spect essential in this research context due to the fact that IOR effects are observed at CTOA longer than approximately $250 \mathrm{~ms}$ has been attributed to that at that time attention has already been disengaged from the cued location (Posner et al., 1985). To overcome this limitation, the alternative cue-back design presents an intervening event (the cue-back) at central fixation during the CTOA interval. This cue-back does not share any characteristic with either the cue or the target and plays the only role of attracting attention away from the cue to ensure that it has been reoriented away from the cued location. With this design, we analyzed differences between cueing conditions in power changes from -3500 to $1000 \mathrm{~ms}$ relative to button press (see methods section), and we found an IOR-related shift from desynchronization to synchronization within the 
145 and $155 \mathrm{~ms}$ posterior to response execution, whereas no power changes between cueing conditions were observed in the interval between the cue and the cue-back, or between the cueback and the target in any frequency band (see Results section). Consequently, it could be concluded that no response effects were observed to either the cue or the cue-back presentation in the present results.

Following the interpretations offered by Pastótter et al. (2008) of their beta changes, and taking into account that in the present study different findings were observed depending on the visual field, the synchronization observed in beta band in the present study could be interpreted as a deactivation of motor areas just after the response was executed to targets appearing at spatially cued locations in the LVF. In the UVF, as it is discussed in the following lines, the results in beta band were different suggesting different underlying mechanisms.

\section{UVF. Beta band $(26-28 \mathrm{~Hz})$}

The only differences between uncued and location-cued trials in the upper visual field were observed around response execution in the beta band. Specifically, a decrease in desynchronization was observed at contralateral postcentral electrodes between 26 and $28 \mathrm{~Hz}$ and from -50 to $20 \mathrm{~ms}$ relative to button press. Beta band rhythms display a desynchronization during movement that has been repeatedly observed in previous studies, and generally assumed to index motor cortical activity participating in movement execution (Pfurtscheller et al., 1996a; Jurkiewicz et al., 2006; Engel and Fries, 2010). The decreased desynchronization observed under spatial cueing in the present study may suggest a late reduction of motor activity when responding to targets appearing at cued locations.

The explanation of such late reduction (or inhibition) of motor activity observed in the beta rhythm in the UVF could be associated with the vertical asymmetries observed in many studies employing attention tasks, including two previous studies in the IOR context (Amenedo et al., 2014; Gutierrez-Domínguez et al., 2014) as discussed below.

In the research context of the present results, the visual field has been found to be an important factor affecting IOR effects on visual and response-related ERP activity with the same task design (Amenedo et al., 2014; Gutiérrez-Domínguez et al., 2014). In those studies, when comparing location-cued trials with the uncued trials, the target-locked ERPs showed significant spatial IOR effects that differed between visual fields in several components. On the latency range of visual N1, spatial cueing evoked lower amplitudes in both visual fields. However, the scalp distribution of such effects varied depending on the visual field where the target was presented. In the LVF, differences were larger at parietooccipital sites, whereas in the UVF these differences showed a centro-frontal distribution (see Gutiérrez-Domínguez et al., 2014). These different distribution patterns were interpreted, following previous results on cortical sources of different subcomponents of N1 (Di Russo et al., 2002), as possibly reflecting the activation of different visual processing areas under spatial IOR in LVF (posterior N1) and in UVF (anterior N1), and therefore different mechanisms affected by spatial IOR.

Moreover, at the rising part of P3, two different location cueing effects were observed (Amenedo et al.,2014; Gutiérrez-Domínguez et al., 2014). While in the LVF an Nd was apparent at posterior electrode sites, in the UVF location cueing elicited a Pd at anterior electrode sites. These results were interpreted in the context of vertical asymmetries observed in visual attention tasks. In this sense, although visual spatial resolution is normally limited by factors ranging from optics to 
neuronal filters in the visual cortex, even in simple visual tasks requiring an overt response (i.e., detection of lines of specific orientation), better performance has been repeatedly observed when attentional focus is directed to stimuli. This effect has been termed attentional resolution, and it is larger when the stimuli are presented in the LVF (Cavanagh et al., 1999). It has been suggested that this LVF advantage in attentional resolution may be partly due to the fact that this visual field is represented in the upper part of the visual cortex, which is anatomically adjacent to and projects more heavily to the occipital-parietal regions that are often linked to spatial attentional control (Gazzaniga and Ladavas, 1987; Maunsell and Newsome, 1987Posner et al., 1987). Moreover, studies on visuospatial attention have found that spatial attention guided by exogenous orienting (peripheral cues) increases the apparent contrast of visual stimulus (Carrasco et al., 2004; Fuller et al., 2008), and this effect has been found to be greater in the LVF (Fuller et al., 2008). Taking the above into account, the Nd observed in our laboratory in the LVF was interpreted as an N2like effect reflecting a re-focusing of spatial attention into target stimuli appearing in the visual field with higher attentional resolution, and at a location where discrimination processes had been previously inhibited (N1 amplitude reductions observed at posterior electrodes). The positive deflection observed in the UVF under spatial IOR, with a later latency and a more anterior scalp distribution than the $\mathrm{Nd}$ observed in the LVF, was related to the elicitation of an orienting response to select a target stimulus presented at a previously inhibited location in the visual field with less attentional resolution. In this sense, the spatial cueing of targets in the UVF could trigger an anterior P2-like effect that would be related to an extra evaluation of, and/or a conflict resolution in working memory, in trials with targets presented in previously inhibited locations (see Gutiérrez-Domínguez et al., 2014).

Regarding response-related ERP activity, although previous research on lateralized readiness potential (LRP) changes under spatial IOR had concluded that spatial IOR does not affect motor processes (Prime and Ward, 2004, 2006), Amenedo et al. (2014) found significant decreases in the amplitude of response-locked LRP when preparing correct responses to cued targets that were interpreted as reflecting less motor activation during response preparation to those stimuli.

Taking the above into account, the reduction in beta desynchronization observed in the UVF in the present study could be interpreted as indicating a reduction of motor activation, at the 'last moment', to targets appearing at previously cued locations in the visual field with less attentional resolution. The existence of poorer attentional resolution in the UVF, therefore could require more evaluation of these stimuli, and/or some conflict resolution in working memory, which would lead to an active inhibition just at the moment of pressing the button instead of reducing the premovement sensorimotor activation when preparing the responses.

\section{Conclusions}

The present results showed IOR effects on response related oscillatory EEG activity that differed between visual fields, which would suggest that different underlying processes are activated depending on the visual field where the target has to be discriminated and a response has to be executed.

In the LVF, IOR was related to synchronization in the pre-movement bilateral mu band, and in the post-movement contralateral beta band. In the UVF, however, reduced desynchronization in contralateral beta band was observed around response execution. 
In the LVF, where there is more attentional resolution, IOR may be associated with an attentiondriven reduction of sensorimotor processing while preparing the execution of responses to relevant stimuli that are presented at previously cued locations whose processing has been reduced in visual areas. The deactivation of motor areas after response execution observed in this visual field could indicate an effective resolution of the response processing.

However, in the UVF, the absence of oscillatory changes in the mu rhythm along with a decrease in beta desynchronization around response execution might indicate a late (last moment) inhibition of motor activation when responding to spatially cued targets that require more evaluation, and/or whose processing generates conflict resolution in working memory, because they appear in a visual field with less attentional resolution.

\section{Acknowledgments}

This study was supported by grants from the Spanish MICINN (PSI2010-21427), Spanish MINECO (PSI2014-53743-P), and Xunta de Galicia (10PXIB211220PR).

\section{References}

Amenedo E, Pazo-Álvarez P, Cadaveira F (2007) Vertical asymmetries in preattentive detection of changes in motiondirection. Int J Psychophysiol 64(2):184-189.

Amenedo E, Gutiérrez-Domínguez FJ, Mateos-Ruger SM, Pazo- Álvarez P (2014) Stimulus-locked and response-locked ERP correlates of spatial inhibition of return (IOR) in old age. J Psychophysiol 28(3):105-123.

Anderson KL, Ding M (2011) Attentional modulation of the somatosensory mu rhythm. Neuroscience 180:165-180.

Aron AR (2011) From reactive to proactive and selective control: developing a richer model for stopping inappropriate responses. Biol Psychiatry 69:55-68.

Bell AJ, Sejnowski TJ (1995) An information-maximization approach to blind separation and blind deconvolution. Neural Comput 7:1129-1159.

Carrasco M, Ling S, Read S (2004) Attention alters appearance. Nature Neurosci 7:308-313.

Cassim F, Monaca C, Szurhaj W, Bourriez J-L, Defebvre L, Derambure P, Guieu J-D (2001) Does postmovement beta synchronization reflect an idling motor cortex? NeuroReport

\section{2:3859-3863.}

Cavanagh P, He S, Intriligator J (1999) Attentional resolution: the grain and locus of visual awareness. In: Taddei-Ferretti C, Musio C, editors. Neuronal basis and psychological aspects of consciousness. Singapore: World Scientific. p. 41-52. 
Cheyne D, Gaetz W, Garnero L, Lachaux JP, Ducorps A, Schwartz D, Varela FJ (2003) Neuromagnetic imaging of cortical oscillations accompanying tactile stimulation. Brain Res Cogn Brain Res 17 (3):599-611.

Delorme A, Makeig S (2004) EEGLAB: an open source toolbox for analysis of single-trial EEG dynamics including independent component analysis. J Neurosci Methods 134:9-21.

Di Russo F, Martínez A, Sereno MI, Pitzalis S, Hillyard S (2002) Cortical sources of the early components of the visual evoked potential. Hum Brain Mapp 15:95-111.

Doallo S, Lorenzo-Lo' pez L, Vizoso C, Rodríguez Holguin S, Amenedo E, Bara’ S, Cadaveira F (2004) The time course of the effects of central and peripheral cues on visual processing: an event-related potentials study. Clin Neurophysiol 115:199-210.

Engel AK, Fries P (2010) Beta-band oscillations - signaling the status quo? Curr Opin Neurobiol 20:156165 .

Fuller S, Rodríguez RZ, Carrasco M (2008) Apparent contrast differs across the vertical meridian: visual and attentional factors. $\mathrm{J}$ Vis $8(1): 1-16$.

Gaetz W, Cheyne D (2006) Localization of sensorimotor cortical rhythms induced by tactile stimulation using spatially filtered MEG. Neuroimage 30:899-908.

Gaetz W, MacDonald M, Cheyne D, Snead OC (2010). Neuromagnetic imaging of movement-related cortical oscillations in children and adults: age predicts post-movement beta rebound. Neuroimage 51:792-807.

Gazzaniga MS, Ladavas E (1987) Disturbances in spatial attention following lesion or disconnection or the right parietal cortex. In: Jeannerod M, editor. Neurophysiological and neuropsychological aspects of visual neglect, vol. 45. Amsterdam: Elsevier Science Publishing. p. 203-213.

Gutiérrez-Domínguez FJ, Pazo- Álvarez P, Doallo S, Fuentes LJ, Lorenzo-López L, Amenedo E (2014) Vertical asymmetries an inhibition of return: effects of spatial and non-spatial cueing on behavior and visual ERPs. Int J Psychophysiol 91(2):121-131.

Haegens S, Hndel BF, Jensen O (2011) Top-down controlled alpha band activity in somatosensory areas determines behavioral performance in a discrimination task. J Neurosci 31 (14):5197-5204.

Hari R, Salmelin R (1997) Human cortical oscillations: a neuromagnetic view through the skull. Trends Neurosci 20:44-49.

Hommel B (2011) The Simon effect as a tool and heuristic. Acta Psychol 136:189-202.

Hopfinger JB, Mangun GR (1998) Reflexive attention modulates processing of visual stimuli in human extrastriate cortex. Psychol Sci 1998(9):441-447.

Hummel F, Andres F, Altenmuller E, Dichgans J, Gerloff C (2002)Inhibitory control of acquired motor programmes in the human brain. Brain 125:404-420.

Huster RJ, Enriquez-Geppert S, Lavallee CF, Falkenstein M, Herrmann CS (2013) Electroencephalography of response inhibition tasks: functional networks and cognitive contributions. Int J Psychophysiol 87:217-233. 
Ivanoff J, Klein RM (2001) The presence of nonresponding effector increases inhibition of return. Psychon Bull Rev 8(2):307-314.

Ivanoff J, Klein RM, Lupia' ñ ez J (2002) Inhibition of return interacts with the Simon effect: an omnibus analysis and its implications. Att Percept Psychophys 64(2):318-327.

Jones SR, Kerr CE, Wan Q, Pritchett DL, Hamalainen M, Moore CI (2010) Cued spatial attention drives functionally relevant modulation of the mu rhythm in primary somatosensory cortex. J Neurosci 30(41):13760-13765.

Jurkiewicz MT, Gaetz WC, Bostan AC, Cheyne D (2006) Postmovement beta rebound is generated in motor cortex: evidence from neuromagnetic recordings. NeuroImage 32:1281-1289.

Karim AK, Kojima H (2010) The what and why of perceptual asymmetries in the visual domain. Adv Cogn Psychol 15 (6):103-115.

Kingstone A, Pratt J (1999) Inhibition of return is composed of attentional and oculomotor processes. Percept Psychophys 61 (6):1046-1054.

Klein RM (2000) Inhibition of return. Trends Cogn Sci 4(4):138-147.

Lee TW, Girolami M, Sejnowski T (1999) Independent Component Analysis using an extended infomax algorithm for mixed subgaussian and supergaussian sources. Neural Comput 11:417-441.

Leocani L, Toro C, Manganotti P, Zhuang P, Hallett M (1997) Eventrelated coherence and event-related desynchronization/ synchronization in the $10 \mathrm{~Hz}$ and $20 \mathrm{~Hz}$ EEG during self-paced movements. Electroencephalogr Clin Neurophysiol 104:199-206.

Lupiáñez J, Klein RM, Bartolomeo P (2006) Inhibition of return: twenty years after. Cogn Neuropsychol 23(7):1003-1014.

Makeig S, Onton J (2012) ERP features and EEG dynamics: an ICA perspective. In: Kappenman Emily S, Luck Steven J, editors. The Oxford handbook of event-related potential components. Oxford University Press.

Maunsell JH, Newsome WT (1987) Visual processing in monkey extrastriate cortex. Ann Rev Neurosci 10:363-401.

Mazaheri A, Niewenhuis ILC, van Dijk H, Jensen O (2009) Prestimulus alpha and mu activity predicts failure to inhibit motor responses. Hum Brain Mapp 30:1791-1800.

McDonald JJ, Ward LM, Kiehl KA (1999) An event-related brain potential study of inhibition of return. Percept Psychophys 61 (7):1411-1423.

Neuper C, Pfurtscheller G (2001) Event-related dynamics of cortical rhythms: frequency-specific features and functional correlates. Int J Psychophysiol 43:41-58.

Oldfield RC (1971) The assessment and analysis of handedness: the Edinburgh inventory. Neuropsychologia 9:97-113.

Parkes LM, Bastiaansen MCM, Norris D (2006) Combining EEG and fMRI to investigate the postmovement beta rebound. NeuroImage 29:685-696. 
Pastotter B, Hanslmayr S, Bauml KH (2008) Inhibition of return arises from inhibition of response processes: an analysis of oscillatory beta activity. J Cogn Neurosci 20(1):65-75.

Pfurtscheller G (2001) Functional brain imaging based on ERD/ERS. Vision Res 41:1257-1260.

Pfurtscheller G, Berghold A (1989) Patterns of cortical activation during planning of voluntary movement. Electroencephalogr Clin Neurophysiol 72(3):250-258.

Pfurtscheller G, Lopes da Silva FH (1999) Event-related EEG/MEG synchronization and desynchronization: basic principles. Clin Neurophysiol 110(11):1842-1857.

Pfurtscheller G, Flotzinger D, Neuper C (1994) Differentiation between finger, toe and tongue movement in man based on $40 \mathrm{~Hz}$ EEG. Electroencephalogr Clin Neurophysiol 90:456-460.

Pfurtscheller G, Stanca' k A, Neuper C (1996a) Post-movement beta synchronization. A correlate of an idling motor area? Electroencephalogr Clin Neurophysiol 98:281-293.

Pfurtscheller G, Stanca' k A, Neuper C (1996b) Event-related synchronization (ERS) in the alpha band an electrophysiological correlate of cortical idling: a review. Int J Psychophysiol 24:39-46.

Pfurtscheller G, Neuper Ch, Andrew C, Edlinger G (1997) Foot and hand area mu rhythms. Int J Psychophysiol 26:121-135.

Pfurtscheller G, Graimann B, Huggins JE, Levine SP, Schuh LA (2003) Spatiotemporal patterns of beta desynchronization and gamma synchronization in corticographic data during self-paced movement. Clin Neurophysiol 114:1226-1236.

Pfurtscheller G, Neuper C, Brunner C, Lopes da Silva FH (2005) Beta rebound after different types of motor imagery in man. Neurosci Lett 378:156-159.

Pfurtscheller G, Brunner C, Schlogl A, Lopes da Silva FH (2006) Mu rhythm (de)synchronization and EEG single-trial classification of different motor imagery tasks. NeuroImage 31:153-159.

Pineda JA (2005) The functional significance of mu rhythms: translating "seeing" and "hearing" into “doing”. Brain Res Rev 50:57-68.

Pogosyan A, Doyle Gaynor L, Eusebio A, Brown P (2009) Boosting cortical activity at beta-band frequencies slows movement in humans. Curr Biol 19:1637-1641.

Posner MI, Cohen Y (1984) Components of visual orienting. In:Bouma H, Bonwhuis D, editors. Attention and performance X:control of language processes. Hillsdale, N. J.: Erlbaum. p. 551-556.

Posner MI, Rafal RD, Choate LS, Vaughan J (1985) Inhibition of return: neural basis and function. Cogn Neuropsychol 2:211-228.

Posner MI, Walker JA, Friedrich FA, Rafal RD (1987) How do the parietal lobes direct covert attention? Neuropsychologia 25:135-145.

Prime DJ, Ward LM (2004) Inhibition of return from stimulus to response. Psychol Sci 15(4):272-276.

Prime DJ, Ward LM (2006) Cortical expressions of inhibition of return. Brain Res 1072(1):161-174.

Prime D, Visser TA, Ward LM (2006) Reorienting attention and inhibition of return. Percept Psychophys 68(8):1310-1323. 
Rezec AA, Dobkins KR (2004) Attentional weighting: a possible account of visual field asymmetries in visual search? Spat Vis 17:269-293.

Ro T, Pratt J, Rafal RD (2000) Inhibition of return in saccadic eye movements. Exp Brain Res 130(2):264268.

Roach BJ, Mathalon DH (2008) Event-related EEG time-frequency analysis: an overview of measures and an analysis of early gamma band phase locking in schizophrenia. Schizophr Bull 34 (5):907926.

Salmelin R, Hari R (1994) Spatiotemporal characteristics of sensorymotor MEG rhythms related to thumb movement. Neuroscience 60:537-550.

Salmelin R, Hamalainen M, Kajola M, Hari R (1995) Functional segregation of movement-related rhythmic activity in the human brain. Neuroimage 2:237-243.

Samuel AG, Kat D (2003) Inhibition of return: a graphical metaanalysis of its time course and an empirical test of its temporal and spatial properties. Psychon Bull Rev 10(4):897-906.

Spence C, Lloyd D, McGlone F, Nichols MER, Driver J (2000). Inhibition of return is supramodal: a demonstration between all possible pairings of vision, touch and audition. Exp Brain Res

$134: 42-48$.

Stanck A, Pfurtscheller G (1996) The effects of handedness and type of movement on the contralateral preponderance of 1-rhythm desynchronization. Electroencephalogr Clin Neurophysiol 99:174182.

Swann N, Tandon N, Canolty R, Ellmore TM, McEvoy LK, Dreyer S, DiSano M, Aron AR (2009) Intracranial EEG reveals a time- and frequency-specific role for R.J. the right inferior frontal gyrus and primary motor cortex in stopping initiated responses. J Neurosci 29:12675-12685.

Swann NC, Cai W, Conner CR, Pieters TA, Claffey MP, George JS, Aron AR, Tandon N (2012) Roles for the pre-supplementary motor area and the right inferior frontal gyrus in stopping action: electrophysiological responses and functional and structural connectivity. NeuroImage 59:28602870 .

Thomas NA, Elias LJ (2011) Upper and lower visual field differences in perceptual asymmetries. Brain Res 1387:108-115. 
Post-print (final draft post-refereeing) 


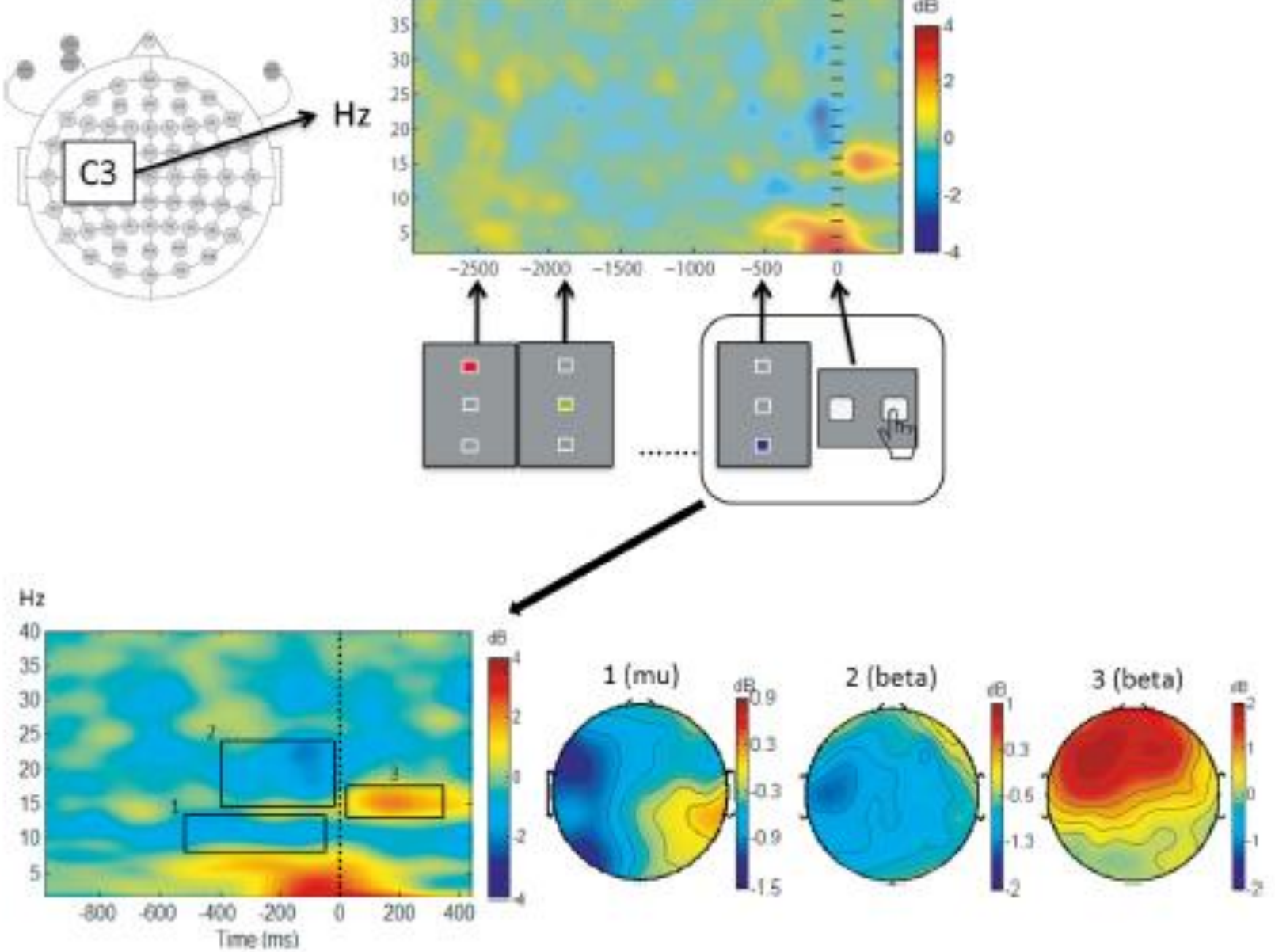

Fig. 2. ERSP image showing roponso-bdoed power changes during a complete uncued tital (-3000 to 500 me rolatwe to burton prex) at C3 abctrode (upperpane). In the bwer panet, the typical osdlatory pathorn obtain od to movoment producton can be observod in a detal of the seme

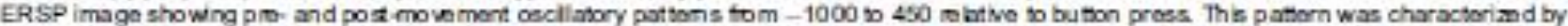
mu desynchronization from taget presentason to the and of the epoch, a beta desynchronizaton from targat to response execution, and a sudden incresso in beta synchonkason attor roponse erocution. 


\section{A}
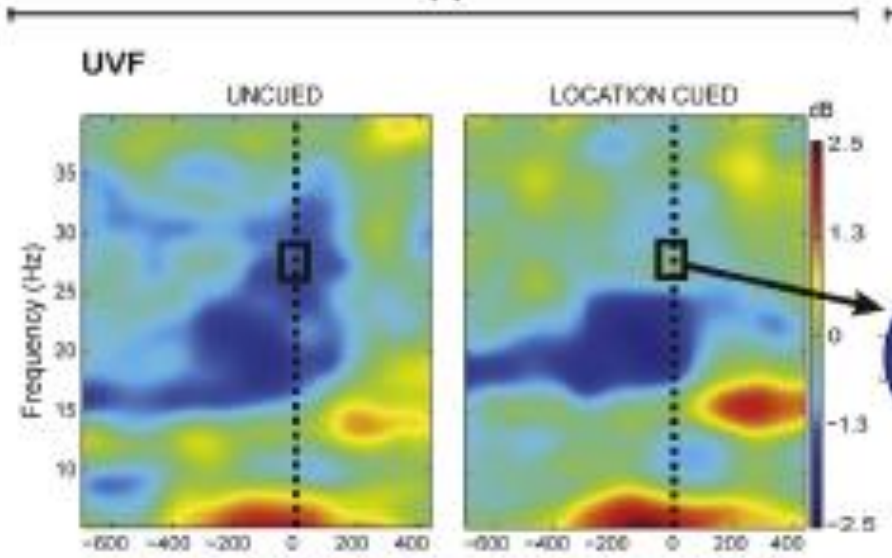

B

B

$\mathrm{C}$

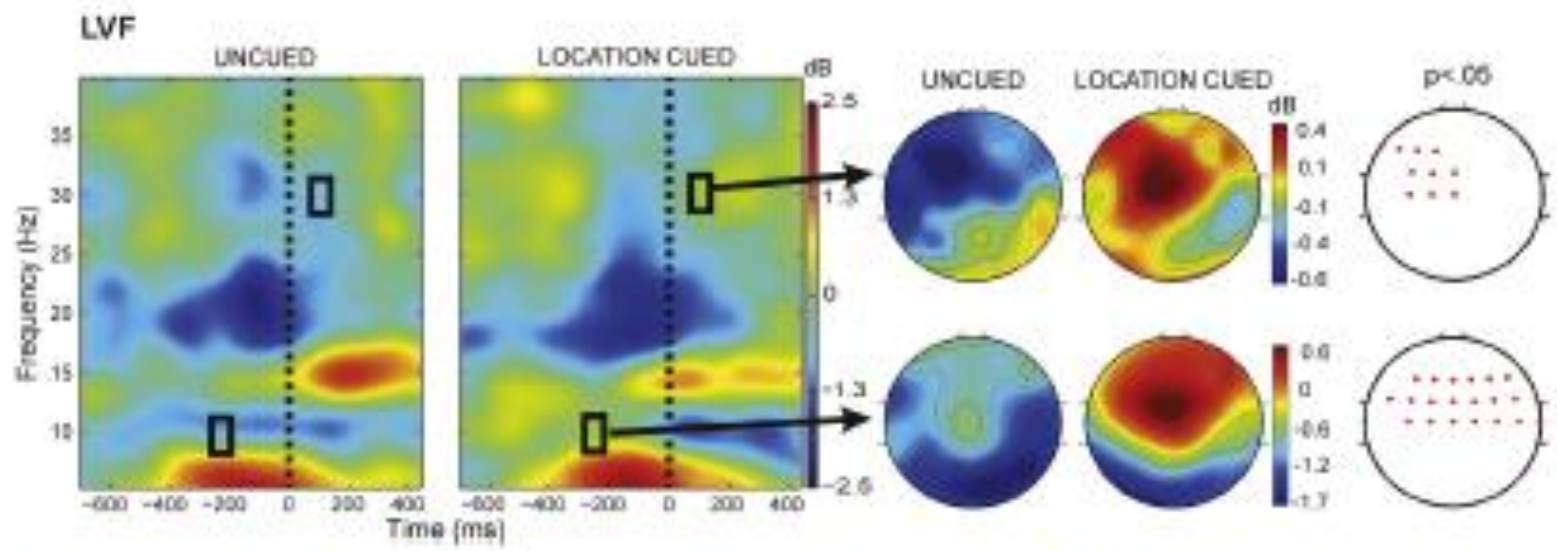

Fig. 3. (A) ERSP imsges showng power changos acros cuoing condtions (lat column: uncuod; right column: locaton cuod) and visual fiolds (upper row: UVF; bwer row: LVF) at the C3 eloctode. Time-fequancy intavals where permutasons with tar correction showed significant dfforences betwoen cueing anditons ate matrod with a black roctangle. Zero point in the tme scales of these imsges (trom -700 to 450 me relative to butson prox) indicatos the butson pross. (B) Topographic maps showing the scalp datibution of the tmo-froquency intarvals where

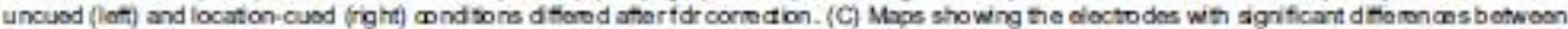
condtions ahar fdr corrocton. 